\title{
Calendar of Short Courses \& Workshops Powder Diffraction June 2015
}

\author{
Gang Wang \\ Research and Development Center for Functional Crystals, Institute of Physics, Chinese Academy of Sciences, No.8 Nansanjie, Zhongguancun, \\ Haidian District, Beijing 100190, China \\ gangwang@iphy.ac.cn
}

6-24 July 2015

2015 Asia-Pacific Edition of HERCULES in Taiwan, Hsinchu, Taiwan, China [Info: http://hercules2015.nsrrc.org.tw/]

\section{3-19 July 2015}

Second Balkan School on Fundamental Crystallography/ Workshop on Magnetic Symmetry and its Application in Magnetic Structure Description, Istanbul, Turkey [Info: http://www.crystallography.fr/mathcryst/istanbul2015.php]

\section{3-24 July 2015}

The São Paulo School of Advanced Sciences (ESPCA) on Recent Developments in Synchrotron Radiation (SyncLight 2015) Campinas, São Paulo, Brazil [Info: http:// pages.cnpem.br/synclight2015/]

\section{July-4 August 2015}

International School of Neutron Science and Instrumentation Erice, Italy [Info: http://www.sonsfpricci. org/sons-school-2015]

\section{5-23 August 2015}

2015 PSI Summer School on Condensed Matter Research (14th edition)-Spectroscopy with Neutrons, Muons, and Photon Lyceum Alpinum, Zuoz, Switzerland [Info: http:// indico.psi.ch/conferenceDisplay.py?confId=3407]

\section{8-31 August 2015}

Electron Crystallography School-ECS2015 Duga Uvala, Croatia [Info: http://www.blogs.uni-mainz.de/ECS2015] 6-18 September 2015 14th Oxford School on Neutron Scattering University of Oxford, Oxford, UK [Info: http://www.oxfordneutronschool. org/]

7-18 September 2015

19th JCNS Laboratory Course-Neutron Scattering 2015 Jülich/Garching, Germany [Info: http://www.fz-juelich.de/ jcns/EN/Leistungen/ConferencesAndWorkshops/LabCourse/ _node.html]

28-30 September 2015

Basic Rietveld Refinement \& Indexing Workshop Newtown Square, Pennsylvania, USA [Info: http://www. icdd.com/education/rietveld-workshop.htm]

28 September-2 October 2015

Autumn School for Neutron Imaging PSI Villigen, Switzerland

More info coming soon

1-2 October 2015

Advanced Rietveld Refinement \& Indexing Workshop Newtown Square, Pennsylvania, USA [Info: http://www. icdd.com/education/rietveld-workshop.htm]

22-26 August 2016

Neutron Powder Diffraction School 2016 Villigen, Switzerland

More info coming soon 